\title{
Induction of renal artery hyperresponsiveness by alpha1- adrenoceptor in hepatorenal syndrome
}

\author{
Xiaogang Zhang ${ }^{1}$, Xinsen $X u^{1}$, Yina Jiang ${ }^{2}$, Jianyu $\mathrm{He}^{3}$, Wenjing Wang ${ }^{1}$, Wei $\mathrm{Li}^{1}$, \\ Xufeng Zhang ${ }^{1}$ and Yi Lv $^{1}$ \\ ${ }^{1}$ Department of Hepatobiliary Surgery, First Affiliated Hospital of Xi'an Jiaotong University College of Medicine, Xi'an 710061, \\ China \\ ${ }^{2}$ Department of Pathology, First Affiliated Hospital of Xi'an Jiaotong University College of Medicine, Xi'an 710061, China \\ ${ }^{3}$ Department of Pharmacology, First Affiliated Hospital of Xi'an Jiaotong University College of Medicine, Xi'an 710061, China \\ Correspondence to: Yi LV, email: luyi169@126.com
}

Keywords: hepatorenal syndrome; $\alpha$ l-adrenoceptor; hyperresponsiveness; renal artery; pathogenesis

Received: February 17, $2017 \quad$ Accepted: June 29, $2017 \quad$ Published: November 25, 2017

Copyright: Zhang et al. This is an open-access article distributed under the terms of the Creative Commons Attribution License 3.0 (CC BY 3.0), which permits unrestricted use, distribution, and reproduction in any medium, provided the original author and source are credited.

\section{ABSTRACT}

Objective: To investigate the potential role of alpha1-adrenoceptor ( $\alpha 1-A R)$ in the pathogenesis of hepatorenal syndrome.

Methods: Hepatorenal syndrome was induced in male rats by intraperitoneal injection of D-galactosamine and orally treatment with $\alpha 1-A R$ antagonist tamsulosin. Hyperresponsiveness of the renal artery contraction was evaluated by the laserDoppler flowmetry and multimyograph system, while renal blood flow (cortical and medullary perfusion) was simultaneously measured. Renal artery ring segment tone was recorded with the myograph system, and concentration-response curves were obtained by cumulative administration of agonists.

Results: This model developed acute renal and liver failure without renal damage in pathology, accompanied by significant hyperresponsiveness of renal artery contraction. After hepatorenal syndrome, plasma concentrations of tumor necrosis factor- $\alpha$ increased by two-fold, and $\alpha 1-A R$ was significantly activated in the renal artery. Concentration-dependent vasoconstriction induced by noradrenaline was significantly decreased in the renal arteries of hepatorenal syndrome rat because of gradually decreased renal blood flow. Administration of tamsulosin prevented renal failure when given before the onset of liver injury, but it had no effect on liver injury by itself.

Conclusion: $\alpha 1-A R$ expression is positively associated with renal vasoconstriction induced by renal artery hyperresponsiveness in HRS. Therefore, $\alpha 1-A R$ may be a potential target in the treatment of HRS.

\section{INTRODUCTION}

Despite considerable advances in the treatment of end-stage liver disease, renal dysfunction remains common and contributes to the morbidity and mortality associated with hepatorenal syndrome (HRS) [1]. It was reported that renal blood flow was reduced in patients with severe acute liver failure (ALF), indicating renal vasoconstriction and pathological change in those patients who develop
HRS [2]. However, the pathogenesis of ALF-induced acute renal failure (ARF) and its underlying molecular mechanisms are poorly understood, although several causal elements, such as splanchnic vasodilatation, reduction of effective arterial volume, and portal hypertension, have been considered as possible pathogenetic factors of renal injury $[3,4]$. Therefore, events independent of systemic hemodynamics may be involved in the pathogenesis of HRS, including renal hemodynamic and/or renal 
vascular resistance changes. In HRS patients, splanchnic vasodilatation and decreased effective arterial volume antedate the development of renal failure, which also activates a variety of compensatory mechanisms (the renin-angiotensin system, sympathetic nervous system, and increased release of antidiuretic hormone etc.), leading to the renal vasoconstriction $[3,5]$. As a result, the decrease of renal blood flow in HRS may be due to the overexpressed renovascular response induced vasoconstriction.

Although renal vasoconstriction is closely related to the development of ARF, it remains unknown about what kinds of receptor types are mainly and functionally responsible for detrimental effects in renovascular hyperresponsiveness [1, 6, 7]. It has been suggested that alpha1-adrenoceptor $(\alpha 1-\mathrm{AR})$ is the functionally relevant adrenoceptor subtype in the renal vasculature of the rat. Additionally, $\alpha 1-\mathrm{AR}$ blockade has been reported to attenuate renal hemodynamic and functional changes induced by renal nerve stimulation and norepinephrine (NE) injection in the kidney [8]. Concurrent administration of $\alpha 1-\mathrm{AR}$ antagonists (prazosin and losartan) mitigated the fall in renal hemodynamics of ARF rats, although the effects of prazosin on renal function were not examined after ischemia reperfusion [9].

In this study, we characterized the renal dysfunction that occurs in the development of ALF induced by D-galactosamine (D-GalN) in an HRS rat model. We aimed to investigate the expression of the $\alpha 1$-AR subtype in renal vascular in this model, and to explore the effect of renal vasoconstriction induced by renovascular hyperresponsiveness via $\alpha 1$-AR modulation in HRS.

\section{RESULTS}

\section{Characterization of a rat model of HRS}

Preliminary experiments showed that approximately $16 \%$ of rats did not develop ARF (creatinine $<50 \mu \mathrm{mol} / \mathrm{L}$ ). The 48 -hour mortality rate was $9 \%$. This time point was chosen because the rats became unwell and the urine volume decreased at around 24-48 hours. This pattern was similar to that of humans, as about $50 \%$ of ALF patients in the intensive care unit develop acute renal dysfunction, with a 10-week mortality rate approaching 90\% and median survival of around 1.7 weeks.

\section{Quantification of renal and hepatic function 48 hours after administration of D-GalN}

Control (Group 1) and D-GalN (Group 2) rats had similar baseline renal and hepatic function. However, a dramatic increase was observed in the D-GalN (Group 2) rats over the controls, of the enzymes ALT (72.03 \pm 16.77 vs. $5954.29 \pm 473.80 \mathrm{IU} / \mathrm{L} ; \mathrm{P}<0.01)$, AST $(83.40$ \pm 13.39 vs. $6943.86 \pm 411.70 \mathrm{IU} / \mathrm{L} ; \mathrm{P}<0.01)$ and serum TBIL $(1.13 \pm 0.34$ vs. $148.70 \pm 33.48 \mu \mathrm{mol} / \mathrm{L} ; \mathrm{P}<0.01)$ at 48 hours after administration of D-GalN (Table 1). The increase in ALT and AST was significantly higher in D-GalN (Group 2) rats with ARF. Serum albumin decreased significantly from $30.80 \pm 3.82$ to $26.30 \pm 2.79$ $\mathrm{g} / \mathrm{L}(\mathrm{P}<0.05)$. As shown in Table 1 , treatment of D-GalN rats with tamsulosin, either before (Group 4) or after (Group 5) administration of D-GalN, had no effect on the severity of liver injury at 48 hours.

${ }^{*} \mathrm{P}<0.05 ;{ }^{* *} \mathrm{P}<0.01$, compared with control.

Serum creatinine $(40.86 \pm 5.52$ vs. $62.57 \pm 5.19$ $\mu \mathrm{mol} / \mathrm{L} ; \mathrm{P}<0.01)$ and BUN $(4.97 \pm 0.80$ vs. $9.21 \pm 1.09$ $\mathrm{mmol} / \mathrm{L} ; \mathrm{P}<0.01)$ in the HRS rat model also increased significantly compared to control. Following D-GalN, a $53 \%$ decrease in Ccr $(1.12 \pm 0.14$ vs. $0.52 \pm 0.05 \mathrm{~mL} /$ $\min ; \mathrm{P}<0.01)$, and an increase in urine creatinine $(1899.7$ \pm 120.15 vs. $2732.86 \pm 138.22 \mu \mathrm{mol} / \mathrm{L} ; \mathrm{P}<0.01)$ were observed. Urinary sodium excretion decreased from 1.17 \pm 0.11 to $0.79 \pm 0.06 \mathrm{mmol} /$ day $(\mathrm{P}<0.05)$. However, FENa increased from $0.58 \% \pm 0.03 \%$ to $1.73 \% \pm 0.11 \%$ $(\mathrm{P}<0.05)$, accompanied by a significant decrease in urine volume $(15.89 \pm 2.04$ vs. $8.11 \pm 1.55 \mathrm{~mL} /$ day $)(\mathrm{P}<$ $0.05)$. These data confirmed that renal function was also impaired in animals that developed ALF.

\section{Histology and quantification of hepatic and renal injury}

The microscopic examination of the livers of D-GalN (Group 2) rats revealed several pathologic changes. Histological evaluation indicated significant focal centrolobular necrosis of hepatocytes, disseminated manifestations of eosinophilic Councilman bodies, enlargement and proliferation of Kupffer cells, and focal accumulations of segmented leukocytes, lymphocytes, plasma cells, and histiocytes, all of these were not observed in the control rats (Figure 1).

Surprisingly, the kidney injury measured by the serum level of BUN and creatinine was not obviously displayed in renal histology in D-GalN (Group 2) rats compared to rats from control group. Except for slight thickening of the basement membranes of glomeruli, renal histopathological examinations at 48 hours did not show any severe lesions after D-GalN treatment in rats with ARF (Figure 2). Furthermore, examination of these kidneys by electron microscopy demonstrated almost normal glomeruli. However, the vacuolar system was slightly more prominent in the proximal tubules, with larger apical vacuoles and prominent vacuoles with flocculent proteinaceous material towards the base of the epithelial cells (Figure 3). In addition, histological changes characterized by minor focal lysis of disarranged smooth muscle cells in renal artery could still be observed in the D-GalN rats. The consequence of this increased prominence of the vacuolar system and focal lysis of smooth muscle cells in the renal artery of D-GalN rats is unknown. 
Table 1: Serum biochemistry 48 hours after injection of D-galactosamine

\begin{tabular}{|c|c|c|c|c|}
\hline Group & ALT (IU/L) & AST (IU/L) & TBIL $(\mu \mathrm{mol} / \mathrm{L})$ & Albumin (g/L) \\
\hline $\begin{array}{l}\text { Control } \\
(\mathrm{n}=10)\end{array}$ & $72.03 \pm 16.77$ & $83.40 \pm 13.39$ & $1.13 \pm 0.34$ & $30.80 \pm 3.82$ \\
\hline $\begin{array}{l}\text { D-GalN } \\
(\mathrm{n}=10)\end{array}$ & $5954.29 \pm 473.80^{* *}$ & $6943.86 \pm 411.70^{* *}$ & $148.70 \pm 33.48^{* *}$ & $26.30 \pm 2.79^{*}$ \\
\hline $\begin{array}{l}\text { tamsulosin controls } \\
(\mathrm{n}=10)\end{array}$ & $74.90 \pm 20.15$ & $80.20 \pm 12.99$ & $1.06 \pm 0.28$ & $30.70 \pm 3.40$ \\
\hline $\begin{array}{l}\text { tamsulosin pre-D-GalN } \\
(\mathrm{n}=10)\end{array}$ & $5877.32 \pm 473.80^{* *}$ & $7003.18 \pm 383.25^{* *}$ & $146.52 \pm 27.58^{* *}$ & $25.90 \pm 2.65^{*}$ \\
\hline $\begin{array}{l}\text { tamsulosin post-D-GalN } \\
(\mathrm{n}=10)\end{array}$ & $6020.46 \pm 473.80^{* *}$ & $6906.86 \pm 353.67^{* *}$ & $150.70 \pm 31.94^{* *}$ & $26.20 \pm 2.28^{*}$ \\
\hline
\end{tabular}

Values are mean $\pm \mathrm{SD}$.

${ }^{*} \mathrm{P}<0.05 ;{ }^{* *} \mathrm{P}<0.01$, compared with control.

\section{$\mathrm{NE}$ induced concentration-dependent vasoconstriction in renal arteries}

In Group 1, NE induced concentration-dependent vasoconstriction in rat renal arteries at a dose from $1 \times$ $10^{-8} \mathrm{~mol} / \mathrm{L}$ to $1 \times 10^{-4.5} \mathrm{~mol} / \mathrm{L}$. The contractile effect occurred 20 seconds after agonist administration. We found that when reaching the maximal contraction, the response declined for about 2 minutes, and did not return to base line easily. $\mathrm{E}_{\max }$ was $81 \% \pm 25 \%$ and PD2 was $6.43 \pm 0.39$ in the NE-induced response curve for Group 1. The concentration-response curve is shown in Figure 4. In Group 2, NE induced contraction in renal arteries in a concentration-dependent manner at a dose from $1 \times 10^{-7.5}$ $\mathrm{mol} / \mathrm{L}^{1}$ to $1 \times 10^{-4.5} \mathrm{~mol} / \mathrm{L}^{1}$. The response took effect 10 seconds after addition of NE, and the contractile curve was significantly elevated. Furthermore, $\mathrm{E}_{\max }$ and PD2 for this group significantly increased from $81 \% \pm 25 \%$ to $154 \%$ $\pm 29 \%(\mathrm{P}<0.01)$, and from $6.43 \pm 0.39$ to $6.70 \pm 0.18(\mathrm{P}$ $<0.05)$, respectively. The concentration-response curve was shown in Figure 4. The results showed a significant difference in NE-induced vasoconstriction in renal arteries between Groups 1 and 2. We found that after addition of D-GalN injection for 48 hours, NE-induced contraction in renal arteries was notably enhanced compared with the vessels of Group 1, suggesting functional upregulation of $\alpha 1-A R$ in Group 2. This clearly indicated that arterial segments from HRS rats yielded an enhanced response to $\mathrm{NE}$, compared with fresh vessel segments from control group rats.

\section{Interaction between renal cortical and medullary perfusion induced by upregulation of $\alpha 1-A R$}

Under baseline condition, the average value of renal cortical LDF (CLDF) was 438.29 $\pm 41.70 \mathrm{PU}$ in control rats. D-GalN administration resulted in a CLDF of 241.46 $\pm 14.96 \mathrm{PU}$, with a decline of $45 \%$ from the control group (Figure 5), and a significant difference between control and HRS rats was observed $(\mathrm{P}<0.01)$. The baseline value of renal medullary LDF (MLDF) was $90.97 \pm 8.68 \mathrm{PU}$ in Group 1. Forty-eight hours after D-GalN administration, MLDF decreased to $87.93 \pm 10.34$ PU (5\% decline), which did not represent a significant difference between Groups 1 and 2 (Figure 6).

In a separate series of experiments, tamsulosin was (pre or post) given to control rats and rats with D-GalNinduced HRS, and renal blood perfusion (CLDF + MLDF) was monitored as previously described. There were no significant differences in CLDF and MLDF between Group 1 and Group 3, indicating that renal baseline hemodynamic variables were not significantly altered by tamsulosin treatment alone. However, the tamsulosin pretreatment (Group 4) blunted the CLDF value (328.23 \pm 17.67 PU vs. $241.46 \pm 16.96 \mathrm{PU} ; \mathrm{P}<0.01)$, but it did not affect MLDF (84.82 $\pm 7.51 \mathrm{PU}$ vs. $87.04 \pm 7.31 \mathrm{PU})$, compared with Group 2 (D-GalN). In contrast, injection with tamsulosin at 36 hours post administration of D-GalN (Group 5) had no effect on either CLDF or MLDF, compared with injection with D-GalN only (Group 2).

\section{TNF- $\alpha$ level in plasma}

TNF- $\alpha$ serum concentration was shown in Figure 7, which was significantly higher in the group that received D-GalN only (Group 2) than in the control group (Group 1). TNF- $\alpha$ increased 1 hour after administration, increased further at 2 hours, and remained elevated for 24 hours compared with the sham group. Changes in serum concentration of TNF- $\alpha$ in tamsulosin-treated animals were undetectable (data not shown). In addition, plasma TNF- $\alpha$ levels in D-GalN only (Group 2) rats were 
not significantly different from rats received tamsulosin pretreatment (Group 4; P>0.05).

\section{Expression of alpha 1 adrenergic receptor}

Western blot was used to evaluate $\alpha 1$-AR expression in different treatment groups, compared with control rats (Figure 8). Forty-eight hours after stimulation with $\mathrm{D}-\mathrm{GalN}$, the $\alpha 1$-AR protein level was decreased in the tamsulosin pre-D-GalN group (Group 4) relative to the D-GalN only group (Group 2). Similarly, a decline in $\alpha 1-A R$ expression was also observed in the tamsulosin post-treatment group (Group 5) compared with Group 2. However, no difference in $\alpha 1-\mathrm{AR}$ protein expression was observed between the two combination treatment groups (Group 4 and Group 5). These results also suggested that $\alpha 1-A R$ expression in the renal artery was increased by ALF induction. In addition, from the immunohistochemistry study, the immunostaining of $\alpha 1$-AR was negative in the kidney from control group, while it was positive in the HRS rats (Figure 9).

\section{DISCUSSION}

A few studies have explored the pathologic mechanisms of renal hypoperfusion in HRS. In our HRS rat model, typical characteristics of ALF, including hepatocellular injury, necrosis of hepatocytes, and inflammatory reaction, were observed. A significant higher TNF- $\alpha$ plasma level, the concentration of which is known to be a predictor of HRS after ALF, was observed 1 hour and 24 hours after administration of D-GalN in our study [10-12]. D-GalN was demonstrated to induce the infiltration of leukocytes and liver cell injury, further releasing proinflammatory cytokines [13, 14]. These cytokines possibly aggravated kidney and renal blood vessel injury, and promoted asystemic inflammatory in response to renal vessel injury $[10,15]$. Therefore, the ALF-induced renal injury by D-GalN was probably due to the excessive inflammatory responses in the HRS model $[10]$.

The biochemical events leading to hepatocellular necrosis and liver dysfunction following administration

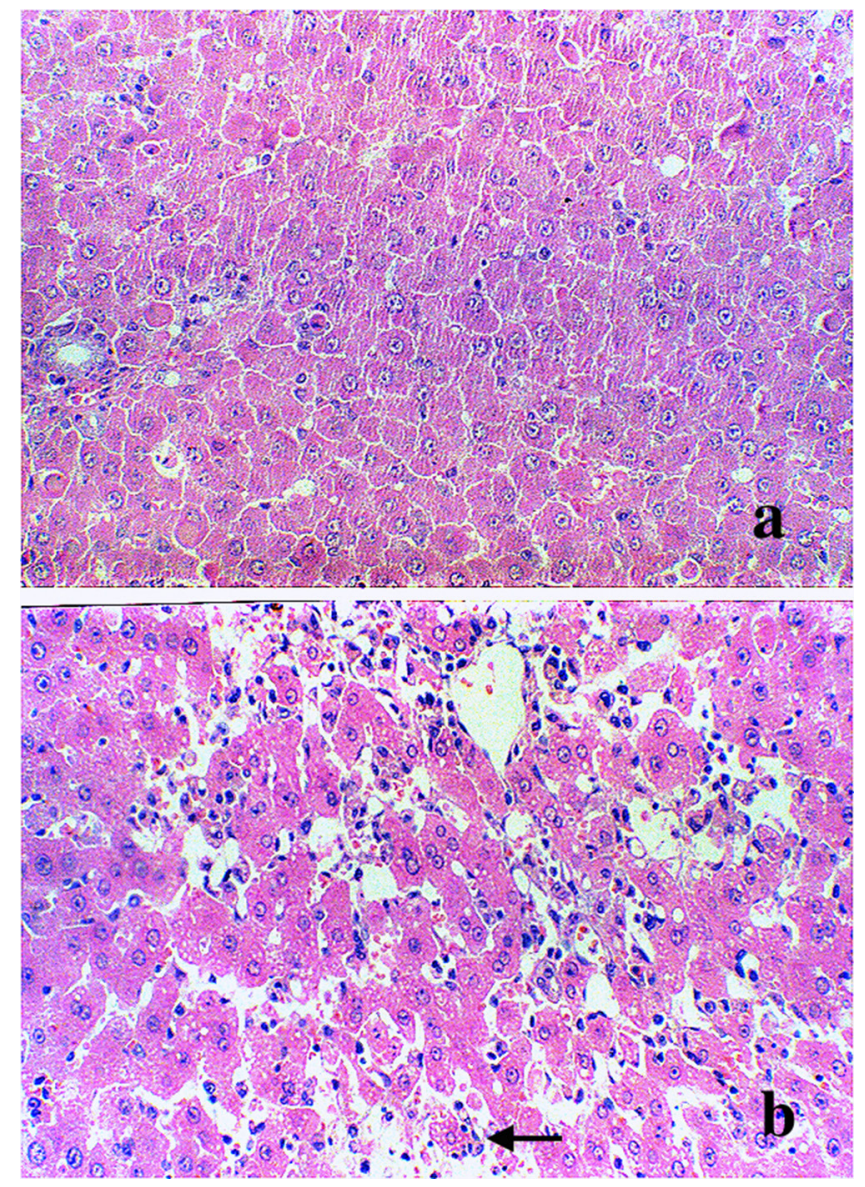

Figure 1: Morphological changes in the liver. Light micrograph (original magnification $\times 200$ ) of liver from (a) control rats and (b) $1200 \mathrm{mg} / \mathrm{kg}$ D-GalN injected rats at 48 hours. Hematoxylin and eosin stained section indicated dilatation of central vein and hepatic sinusoid. Vacuoles appeared in the cytoplasm of hepatocytes (black arrow). Hepatocellular necrosis could also be found in the D-GalN animal's liver. 


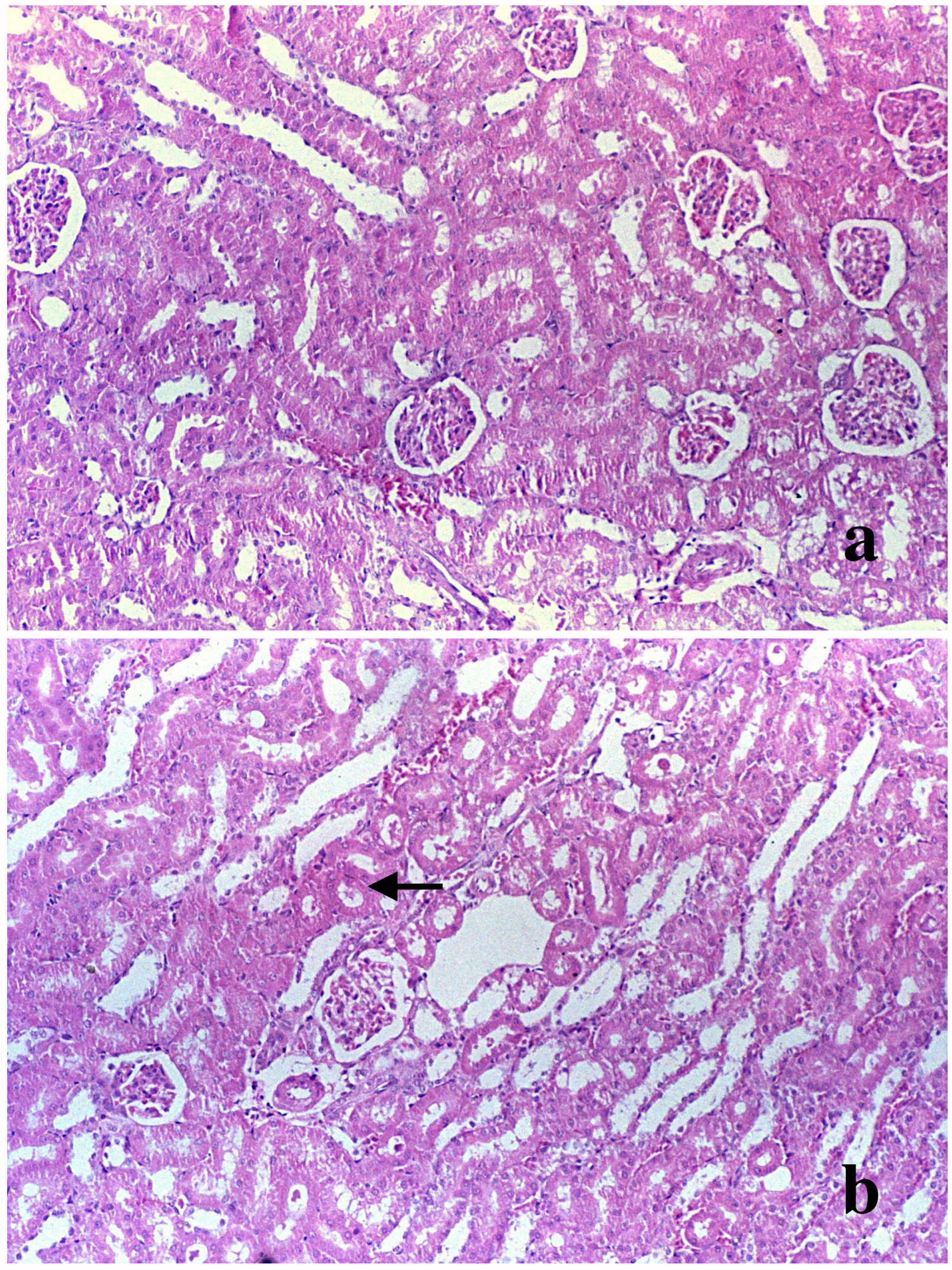

Figure 2: Morphological changes in the kidney. Light micrograph (original magnification $\times 400$ ) of kidney from (a) control rats and (b) $1200 \mathrm{mg} / \mathrm{kg}$ D-GalN injected rats at 48 hours. Hematoxylin and eosin stained section of kidneys showed no abnormality of the renal cortex or medulla in the D-GalN animals, except for slight thickening of the basement membranes of renal tubule (black arrow). 
of D-GalN have been investigated in depth over the years, but the characterization and pathogenesis of renal injury and dysfunction have rarely been explored $[16,17]$. In our study, interestingly, despite the development of renal dysfunction, the kidney structure was histologically normal. The reduction of $\mathrm{UNa}$ in D-GalN-treated rats with HRS implied that tubular function was preserved; however, this may have been influenced by the decreased food and water intake of animals with liver failure. A significant reduction of CLDF and MLDF in kidneys in our model confirmed that renal hypoperfusion occurred independently of any changes in hyperdynamic circulation, and was secondary to renal vasoconstriction. It occurred despite an increase in cardiac output and systemic vasodilatation, as observed in other studies [6, 18, 19]. These major
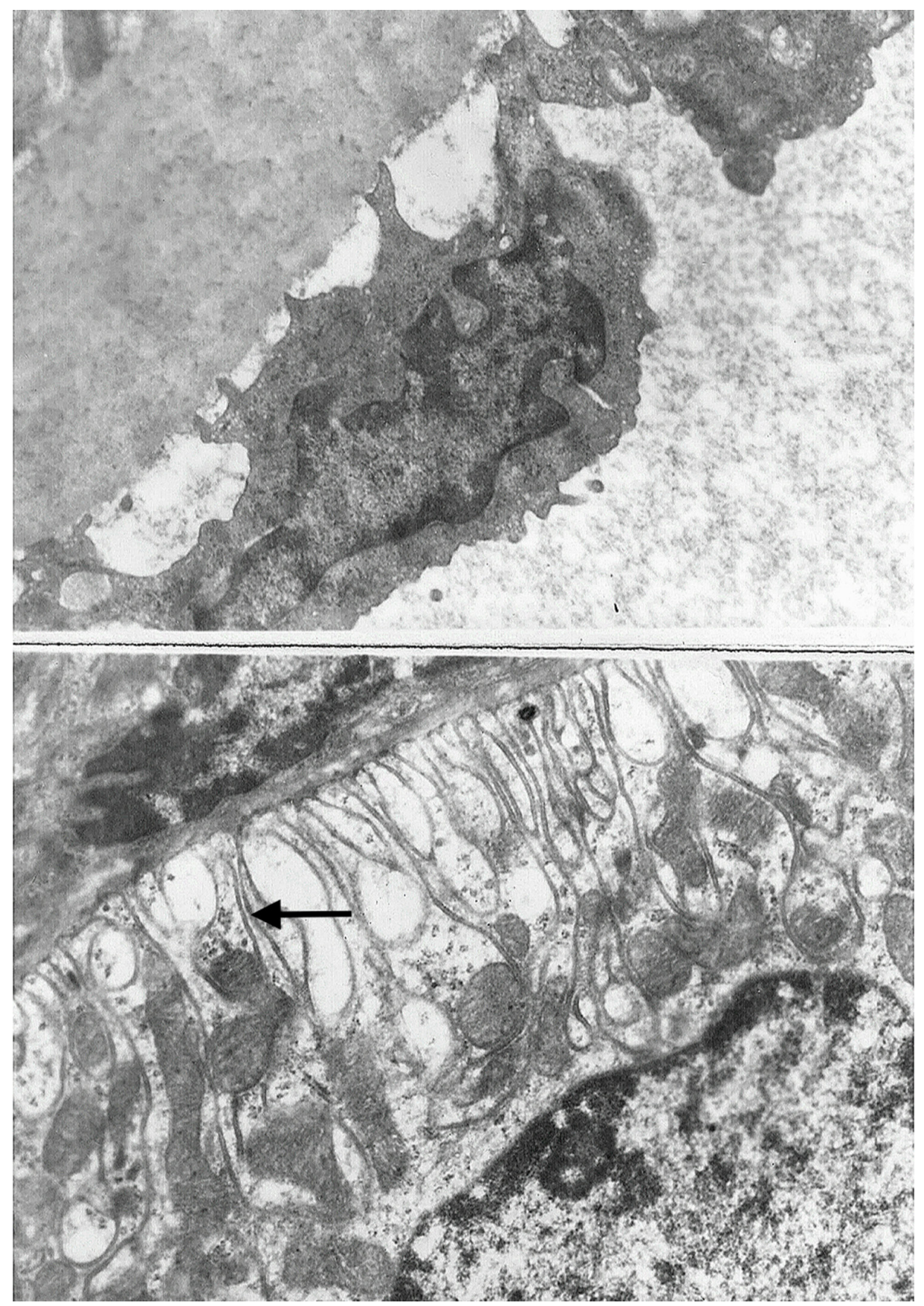

Figure 3: Electron micrograph (original magnification $\times 10000$ ) of (a) renal artery and (b) kidney from $1200 \mathrm{mg} / \mathrm{kg} \mathrm{D-GalN-injected}$ rats at 48 hours. Kidney and renal artery sections were fixed in glutaraldehyde, and stained with uracyl acetate and Reynold's lead citrate. In D-GalN animals, the glomeruli and renal artery were normal. However, in the proximal tubules, the vacuolar system was slightly more prominent, with larger apical vacuoles as well as more prominent vacuoles, with flocculent proteinaceous material towards the base of the epithelial cells (black arrow). 


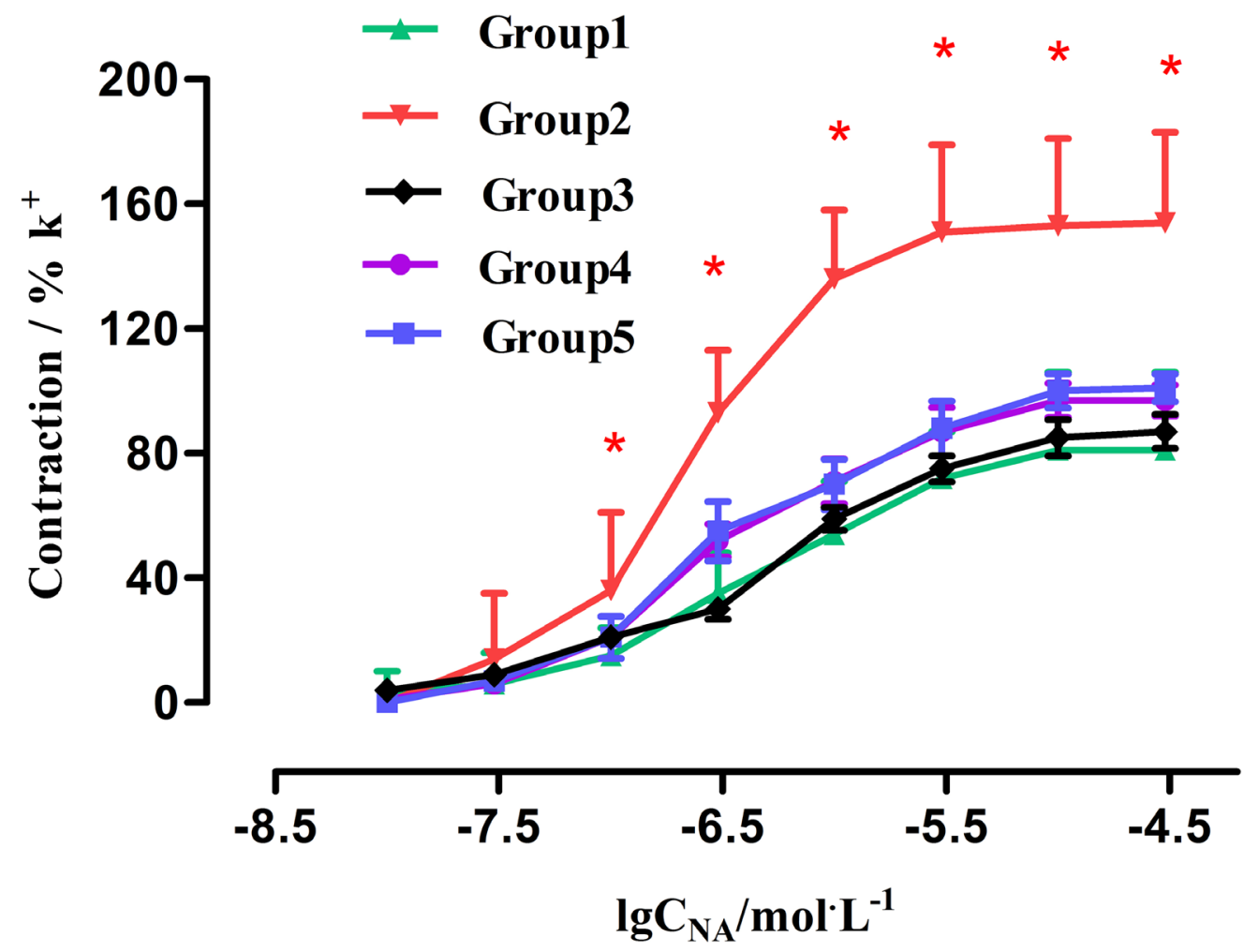

Figure 4: Effect of Tam on concentration-contractile curves induced by NE in rat renal artery. Group1: control $(\mathrm{n}=8)$; Group2: GalN ( $\mathrm{n}=8)$; Group4: Tam pre-D-GalN $(\mathrm{n}=8)$; Group5: Tam post-D-GalN $(\mathrm{n}=8) .{ }^{*} \mathrm{P}<0.01$, compared with control animals.

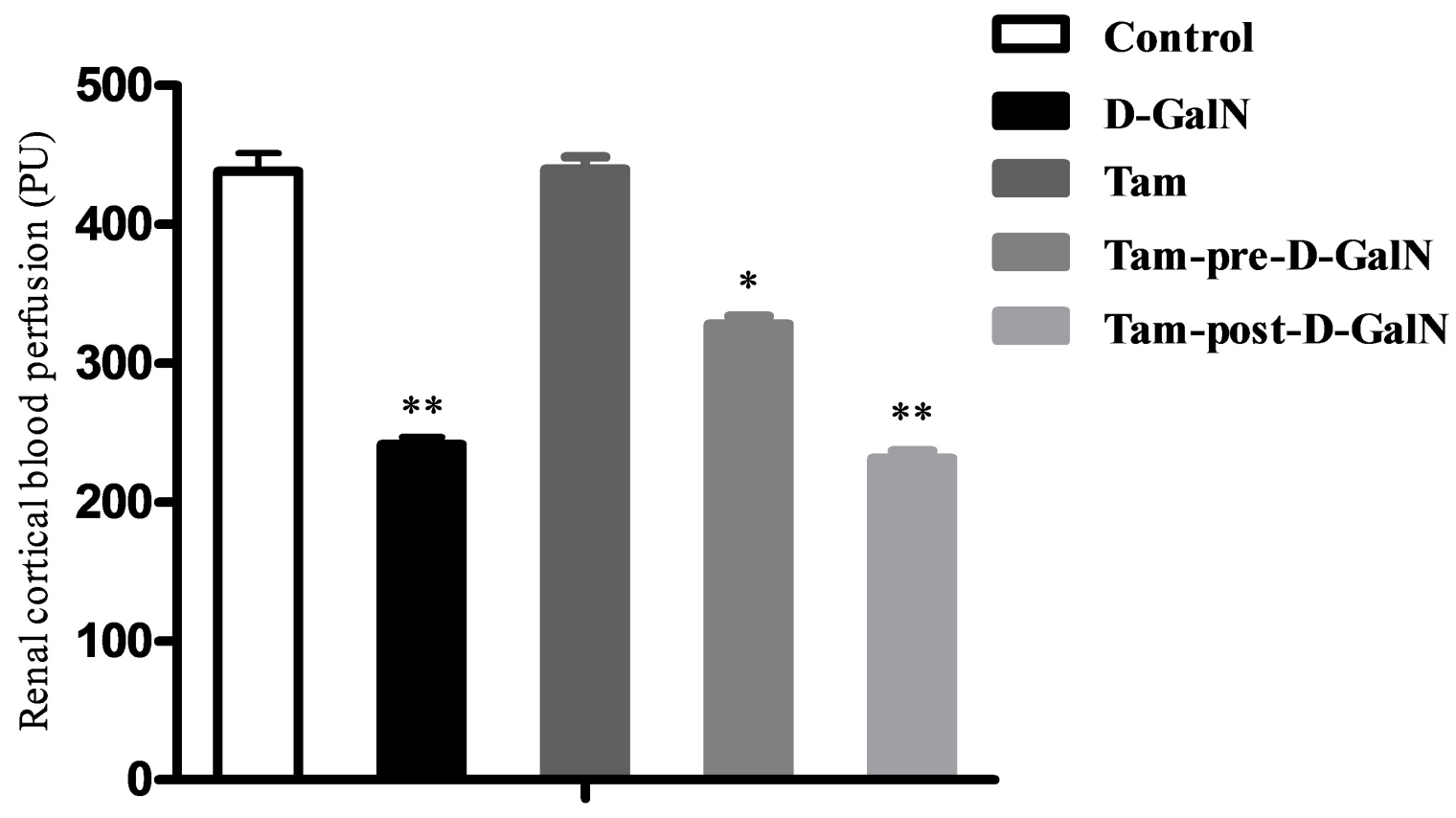

Figure 5: Assessment of renal cortical laser-Doppler flux (CLDF) in D-GalN rats and control rats. Control: CLDF were measured at 48 hours after injection of saline. D-GalN: CLDF were measured at 48 hours after injection of D-GalN. Tam: CLDF were measured at 48 hours after administration of tamsulosin. Tam-pre-D-GalN: animals received tamsulosin 72 hours prior to D-GalN intraperitoneally, and CLDF were measured at 48 hours after administration of D-GalN. Tam-post-D-GalN: animals received tamsulosin 36 hours after D-GalN intraperitoneally, and CLDF were measured at 48 hours after administration of D-GalN. ${ }^{* *} \mathrm{P}<0.01$, compared with control animals. ${ }^{*} \mathrm{P}<0.01$, compared with $\mathrm{D}-\mathrm{GalN}$ animals. 


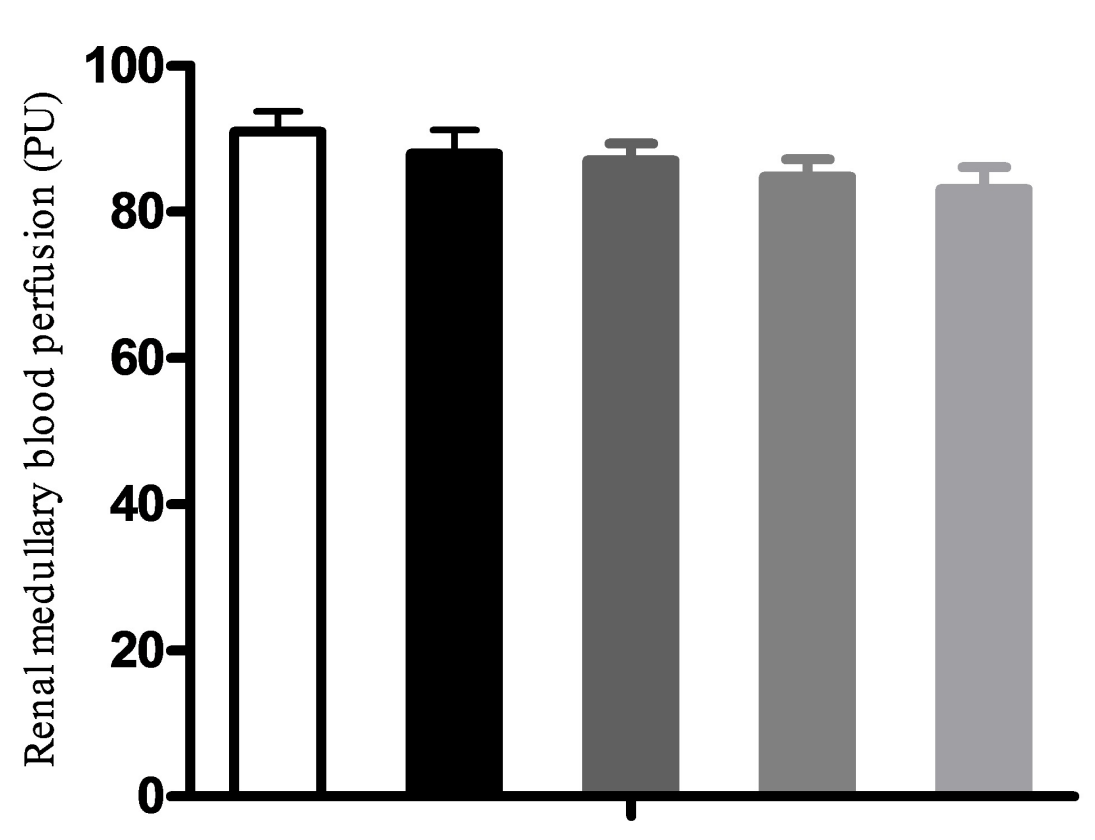

Control

D-GalN

Tam

Tam-pre-D-GalN

Tam-post-D-GalN

Figure 6: Assessment of renal medullary laser-Doppler flux (MLDF) in D-GalN rats and control rats. Control: MLDF were measured at 48 hours after injection of saline. D-GalN: MLDF were measured at 48 hours after injection of D-GalN. Tam: MLDF were measured at 48 hours after administration of tamsulosin. Tam-pre-D-GalN: animals received tamsulosin 72 hours prior to D-GalN intraperitoneally, and MLDF were measured at 48 hours after administration of D-GalN. Tam-post-D-GalN: animals received tamsulosin 36 hours after D-GalN intraperitoneally, and MLDF were measured at 48 hours after administration of D-GalN.

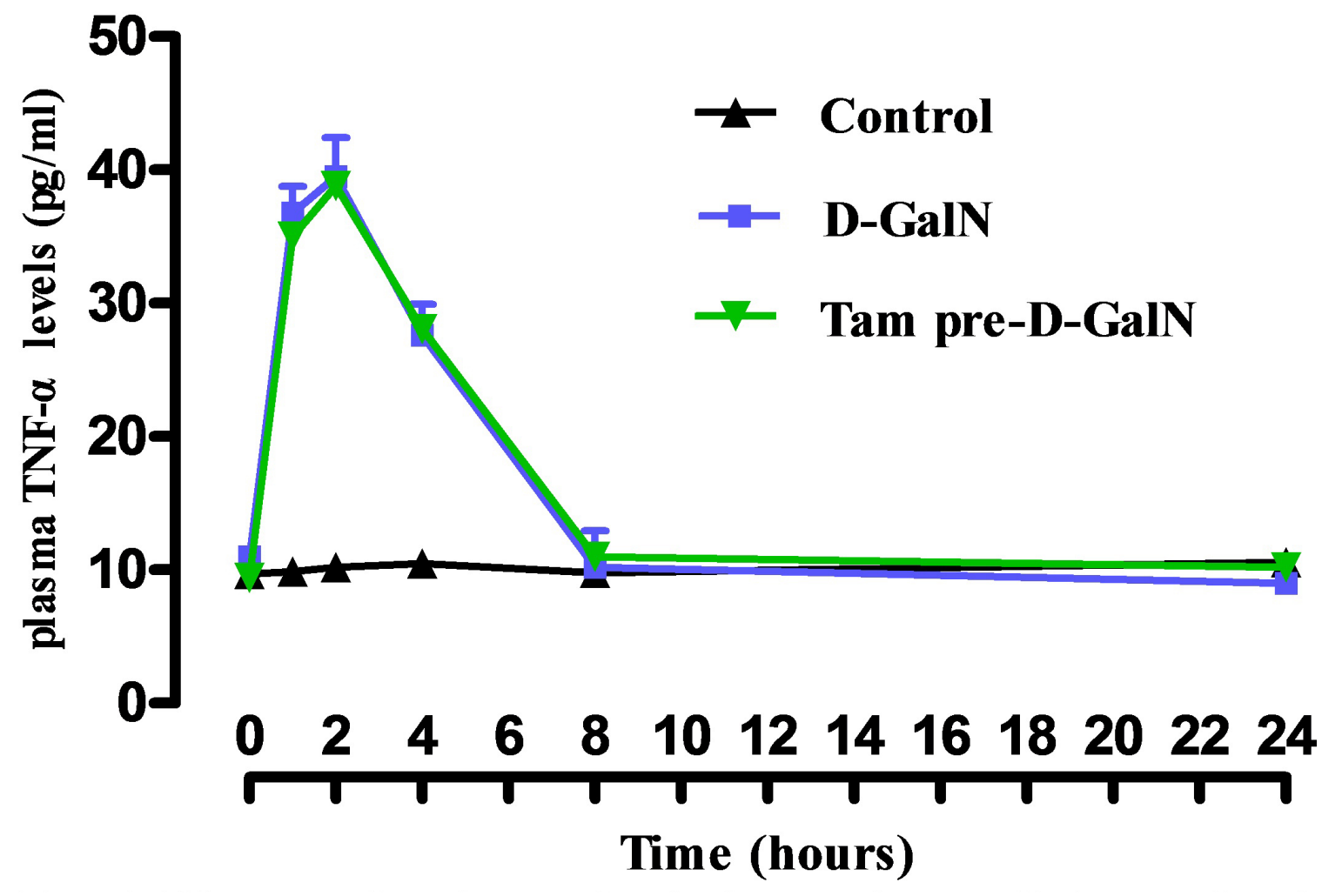

Figure 7: Effects of prior administration of tamsulosin on TNF- $\alpha$ in serum of rats treated with D-GalN. Control: received $2 \mathrm{ml}$ of saline intraperitoneally. D-GalN: were administered $1200 \mathrm{mg} / \mathrm{kg}$ D-GalN intraperitoneally. Tam-pre-D-GalN: received tamsulosin 72 hours prior to D-GalN intraperitoneally. Data were shown as means \pm standard deviations. 
findings indicated that administration of D-GalN to Sprague-Dawley rats caused significant hepatocellular injury, inflammatory response, hyperdynamic circulation, and renal vasoconstriction, which resulted in acute renal failure without irreversible structural renal damage [13, 17]. A previous study demonstrated that D-GalN had no direct toxic effect on cultured LLCPK1 cells, a renal tubular cell line $[10,20]$. These results suggested that local changes in pathophysiology, such as renal vascular response and peripheral vascular dilatation resulting from renal vasoconstriction, rather than from the toxic effect of D-GalN, seemed to contribute to HRS as a result of renal hypoperfusion and receptor dysregulation.

HRS is commonly defined as a purely hemodynamic consequence of liver disease resulting in renal failure [5]. In the settings of decreased peripheral vascular resistance, hypovolemia, and the activation of compensatory mechanisms, the kidneys receive even less blood flow resulting in injury. However, Transplant follow-up data consistently shows that pre-transplant renal dysfunction, regardless of acuity, is associated with increased long term renal and overall survivals [11]. These findings are unexpected if HRS is solely hemodynamic and only a manifestation of the degree of systemic circulatory disorder. Therefore, based on our data, we propose that the renal hypoperfusion in HRS is caused by renovascular hyperresponsiveness via $\alpha 1-\mathrm{AR}$ modulation.

Autoradiographic analyses with specific radioligands demonstrated that the renal $\alpha 1$-ARs were mainly located in the cortex, especially in the proximal tubules, with a lower density in the medulla, playing an important role in controlling renal blood flow and electrolytes [2124]. The effects of ALF on the functional contribution of

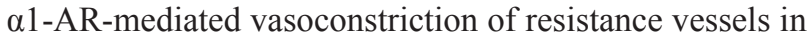
HRS rats have not been fully elucidated. We found that renal arterial hyperresponsiveness was a key factor, which, even if it did not reach shock values, caused simultaneous renal vasoconstriction and renal hypoperfusion with decreased glomerular filtration. In an attempt to identify possible mechanisms by which renal arterial hyperresponsiveness might cause acute renal ischemia, injury, and dysfunction, we used in vitro isometric tension recording on renal artery rings in the HRS rat model. Compared with controls, a higher $\mathrm{E}_{\max }$ was noted with $\alpha 1$ AR in our HRS rat model, with data showing a highly significant increase in renal vascular response during the 48-hour experimental period after ALF induction. Furthermore, administration of the $\alpha 1-\mathrm{AR}$ inhibitor, tamsulosin, significantly suppressed hyperresponsiveness of the renal artery contraction, while administration of tamsulosin post-D-GalN did not affect results, suggesting that tamsulosin did not influence the progress of ALF.

Further evidence supported the effectiveness of for $\alpha 1-\mathrm{AR}$ in the pathogenesis that pre- or post-ischemic treatment with prazosin ameliorated renal dysfunction and tissue injury in ischemia/reperfusion-induced acute renal failure rats $[9,25]$. However, no further studies using highly specific AR receptor antagonists have been carried out in the HRS model or in human HRS, and the role of selective AR antagonists in the treatment of HRS is still conjectural. In our study, a decrease in CLDF value and significant upregulation of the AR receptor in the renal cortex were observed in HRS rats. Interestingly, administration of tamsulosin 72 hours before D-GalN reduced the contribution of AR subtype in mediating adrenergically induced renal vasoconstriction and hypoperfusion. However, no difference in MLDF was detected between HRS rats and pre-/post-tamsulosintreated HRS rats, indicating that neurotransmitters, rather than noradrenaline, may contribute to ALF-induced reductions in MLDF. Previous studies have also found the effect of prazosin on MLDF responses to AR was more difficult to detect than the effect on CLDF responses, simply reflecting the greater variability of receptors in the medulla compared with the cortex [26]. These data, together with findings that tamsulosin prevents the development of renal cortical ischemia, confirmed that
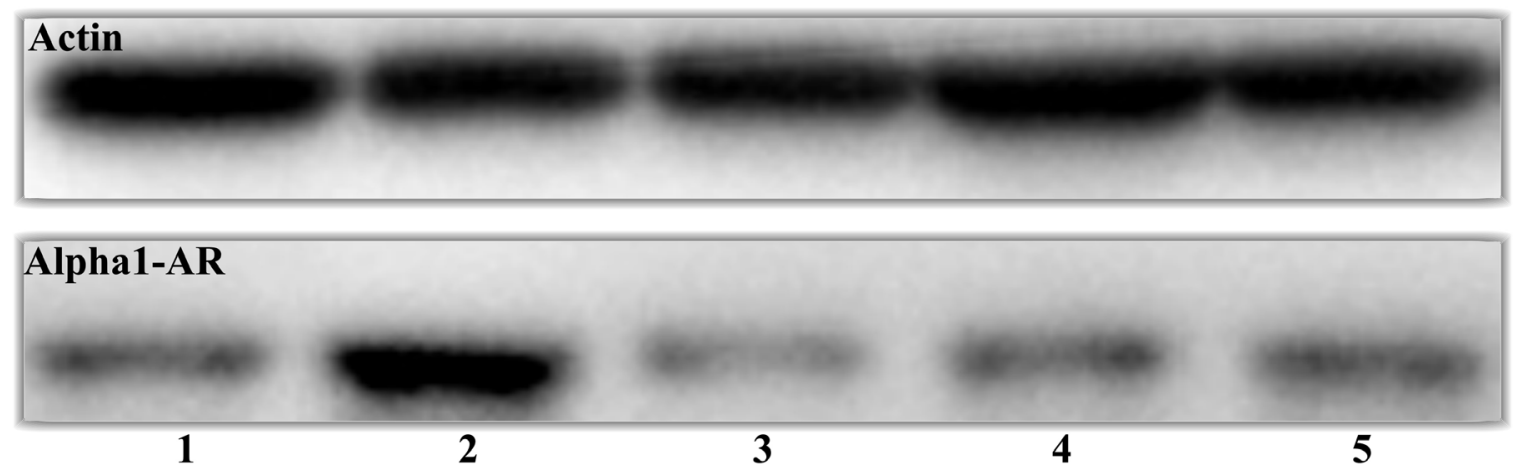

Figure 8: Effect of tamsulosin on $\alpha 1-A R$ in HRS rat renal artery. Group 1: control ( $\mathrm{n}=10$ ); Group 2: $\mathrm{D}-\mathrm{GalN}$ ( $\mathrm{n}=10$ ); Group 3: tamsulosin $(n=10)$; Group 4: tamsulosin pre-D-GalN ( $\mathrm{n}=10)$; Group 5: tamsulosin post-D-GalN $(\mathrm{n}=10)$. Renal artery tissue was lysed and subjected to immunoblot analysis for anti- $\alpha 1-\mathrm{AR}$. Actin was used as control for protein loading. 


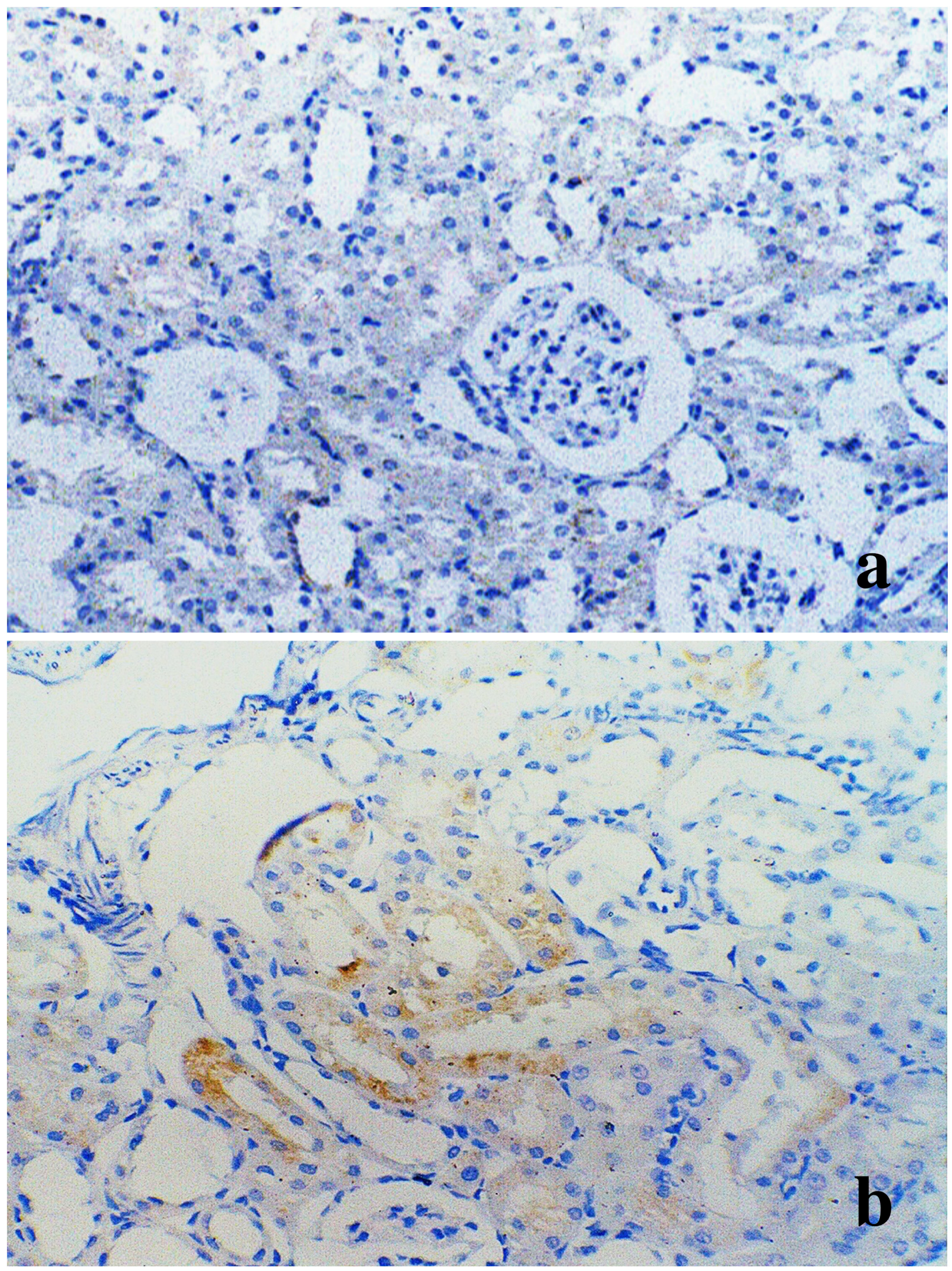

Figure 9: Immunohistochemistry of $\alpha 1-A R$ in kidney from control and HRS rats. (a) Negative immunostaining in renal cortex from control rats (Group 1) (original magnification $\times 200$ ); (b) $\alpha 1$-AR immunoreactivity is observed in proximal tubules from HRS rats (Group 2) (original magnification $\times 400$ ). 
AR played an important role as a determinant of renal perfusion in this HRS model.

In summary, $\alpha 1$-AR-mediated hyperresponsiveness of the renal artery contraction give rise to renal vasoconstriction in HRS rats, which may be a mechanism for HRS. Administration of selective adrenergic agonists altered the upregulation of AR subtypes in mediating adrenergically induced renal vasoconstriction and hypoperfusion.

\section{MATERIALS AND METHODS}

\section{Experimental animals}

The experimental protocol was approved by the local ethical committee for animal research of Xi'an Jiaotong University. Male Sprague-Dawley rats (each weighs around 200-250 g) were obtained from Xi'an Jiaotong Comparative Biology Unit, and kept a lightcontrolled room with a 12 hour light/dark cycle, at a temperature of $19-25^{\circ} \mathrm{C}$, and humidity of approximately $55 \% \pm 5 \%$. Rats were placed individually in polycarbonate metabolic cages with free access to food and water. D-GalN was administered as an intraperitoneal injection of $1200 \mathrm{mg} / \mathrm{kg}(5.5 \mathrm{mmol} / \mathrm{kg})$ in a $200 \mathrm{mg} / \mathrm{mL}$ solution in saline $(\mathrm{pH}$ 6.8). Controls received $2 \mathrm{~mL}$ of saline intraperitoneally. Tamsulosin, a selective $\alpha 1-\mathrm{AR}$ antagonist was administered daily at $0.03 \mathrm{mg} / \mathrm{kg}$ in $1 \%$ sodium carboxymethylcellulose.

The animals were divided into five groups by different treatments: Group 1 (control group, $\mathrm{n}=10$ ) was given $2 \mathrm{~mL}$ of saline intraperitoneally; Group 2 (D-GalN group, $\mathrm{n}=10$ ) was administered $1200 \mathrm{mg} /$ kg D-GalN intraperitoneally; Group 3 (tamsulosin controls, $\mathrm{n}=10$ ) received tamsulosin orally and saline intraperitoneally; Group 4 (tamsulosin pre-D-GalN, n = 10) received tamsulosin orally 72 hours prior to D-GalN intraperitoneally; and Group 5 (tamsulosin post-D-GalN, n $=10$ ) received tamsulosin 36 hours after D-GalN.

\section{Biochemical studies}

Blood was collected from the caudal vein or inferior vena cava and put into the ethylenediamine tetra-acetic acid or plain tubes and centrifuged at $2000 \mathrm{~g}$ for 10 minutes at $4{ }^{\circ} \mathrm{C}$. With plasma or serum, it was then stored at $-80^{\circ} \mathrm{C}$. Quantitative liver and renal function tests, including measurement of levels of aspartate transaminase (AST) and alanine transaminase (ALT), albumin and total bilirubin (TBIL), as well as serum and urine creatinine, and blood urine nitrogen (BUN), were carried out using an automatic analyzer (Hitachi, Japan). Urine sodium concentration (UNa) and plasma sodium concentration (PNa) were determined using a flame photometer (Hitachi, Japan). Creatinine clearance (Ccr) was calculated from urine and serum creatinine values. Fractional excretion of sodium (FENa, \%) was calculated using the formula:
$\mathrm{FENa}=\mathrm{UNaV} /(\mathrm{PNa} \times \mathrm{Ccr}) \times 100$, where $\mathrm{V}$ is 24 -hour urine volume.

\section{Vascular response to stimulation with vasoconstrictor}

The renal artery was immediately removed and carefully cleaned of connective tissue and blood, then cut into 2 or $3 \mathrm{~mm}$ renal arterial rings. These rings were mounted on two L-shaped stainless steel pins in a myograph chamber. One pin was connected to a force displacement transducer (JH-2) to record isometric tension with a MultiMyograph System-610M (Danish Myo Technology A/S, Denmark) attached to a digital converter unit. Another pin was connected to a manual screw, which allowed for fine vascular tone adjustments by varying the distance between the two pins. Measurements were recorded on a computer using a Power Lab Unit (AD Instruments, Oxford, UK). The renal arterial rings were immersed in a temperature-controlled buffer solution $\left(37^{\circ} \mathrm{C}\right)$, which was continuously gassed with a $5 \% \mathrm{CO}_{2}$ in $\mathrm{O}_{2}$ gas mixture, resulting in a stable $\mathrm{pH}$ of 7.4. During an equilibration period of 120 minutes, a baseline tension of $2 \mathrm{mN}$ was adjusted. After 2 hours of equilibration, potassium-rich $(60 \mathrm{mM}) \mathrm{Kreb}$ 's buffer solution was used to determine segment contractile function as a contractile capacity reference. When two reproducible contractions had been achieved, the vessels were used for further experiments. Vascular segment concentration-response curves were obtained by cumulative administration of $\mathrm{NE}$ (10-8-10-4.5 mM). $\mathrm{E}_{\max }$ represented the maximal constriction induced by NE $\left(1 \times 10^{-10}-1 \times 10^{-3} \mathrm{~mol} / \mathrm{L}\right)$ in preconstricted arterial rings. PD2 represented the negative logarithm of the concentration that produced $50 \%$ of the maximal contractile effect.

\section{Cortical and medullary perfusion measurements}

Anesthesia was induced with 5\% isoflurane followed by $2.5 \%$ in $30 \%$ oxygen- $70 \%$ nitrous oxide. After 48 hours of D-GalN or saline administration, the left kidney was exposed by midline abdominal incision. Renal cortical and medullary perfusion were measured with a Perimed FeriFlux System 5000 flowmeter (Sweden), using a microprobe secured with a self-adhesive probe (which reduces the impact of artefactual movement and ensures good optical coupling) applied to a point on the upper left anterior surface of the kidney. The laser-Doppler flux (LDF) signal from the kidney, corresponding to the cortical perfusion, was sampled at $100 \mathrm{~Hz}$ and measured using a time constant of 0.03 seconds.

\section{Histology, immunohistochemistry, and western blotting}

In the end, rats were euthanized by stunning and exsanguination. Liver and kidney samples were fixed in 
$10 \%$ neutral buffered formalin, embedded in paraffin, sectioned, and stained with hematoxylin and eosin for light microscopy examination. To study the vascular microstructure, a transmission electron microscope (TEM, H-600, Hitachi, Japan) was used. The fresh vessels were sectioned at $1 \mathrm{~mm} \times 3 \mathrm{~mm} \times 3 \mathrm{~mm}$ and fixed with $2.5 \%$ glutaraldehyde $+4 \%$ paraformaldehyde in phosphate buffer for 2 hours at $4^{\circ} \mathrm{C}$, and washed with $0.1 \mathrm{M}$ phosphate buffer for 30 minutes. Ultrathin sectioning at 50-70 nm was carried out (LKB-V, Sweden); sections were stained with uranyl acetate and lead hydroxide for 10 minutes, and subsequently examined in the transmission electron microscope.

After homogenizing renal artery tissue, total protein was extracted. A Coomassie brilliant blue assay was used to determine total protein concentration. Proteins were resolved by $10 \%$ sodium dodecyl sulfate polyacrylamidegel, transferred to a nitrocellulose filter membrane using a semidry electrophoretic graphite electrode with a constant current of $1 \mathrm{~mA} / \mathrm{cm}^{2}$ gel for 1 hour, and probed with the diluted specific antibody to $\alpha 1$-AR ( $\beta$-actin was used as an internal standard), followed by horseradish-peroxidase-conjugated secondary antibody. An antibody chromogenic agent (Santa Cruz Biotechnology Inc, USA) was placed on the membrane for the development of a film, and a scanner was used for imaging. Densitometry was used to measure the protein level.

\section{Statistics}

All data were expressed as mean values \pm standard error of the mean. The concentration-effect curves of agonists were fitted to the Hill equation using an iterative, least square method (Graph Pad Prism, San Diego, CA, USA) to provide estimates of maximal contraction $\left(\mathrm{E}_{\max }\right)$ and pEC50 values (negative logarithm of the concentration that produced $50 \%$ of the maximal effect). Two-way analysis of variance (ANOVA) with Dunnett's test posthoc was used for comparisons between all treatment groups. $\mathrm{P}<0.05$ was considered as statistically significant. The comparison of histology scores was analyzed by the Mann-Whitney test.

\section{Author contributions}

Zhang XG: wrote the paper and performed the research; Xu XS: revised the paper; Jiang YN, Wang WJ, He JY, Li W, Zhang XF: performed the research and collected data; Lv Y: designed the research.

\section{ACKNOWLEDGMENTS}

We would like to thank the reviewers and editors for the valuable advices for the paper.

\section{CONFLICTS OF INTEREST}

The authors declare that there is no conflicts of interest regarding the publication of this article.

\section{FUNDING}

This research was supported by the National Natural Science Foundation of China (No. 30600575).

\section{REFERENCES}

1. Gluud LL, Christensen K, Christensen E, Krag A. Systematic review of randomized trials on vasoconstrictor drugs for hepatorenal syndrome. Hepatology. 2010; 51: 576-84. W.

2. Lata J. Hepatorenal syndrome. World J Gastroenterol. 2012; 18: 4978-84. https://doi.org/10.3748/wjg.v18.i36.4978.

3. Venkat D, Venkat KK. Hepatorenal syndrome. South Med J. 2010; 103: 654-61. https://doi.org/10.1097/ SMJ.0b013e3181e07751.

4. Barreto R, Fagundes C, Guevara M, Sola E, Pereira G, Rodriguez E, Graupera I, Martin-Llahi M, Ariza X, Cardenas A, Fernandez J, Rodes J, Arroyo V, et al. Type-1 hepatorenal syndrome associated with infections in cirrhosis: natural history, outcome of kidney function, and survival. Hepatology. 2014; 59: 1505-13. https://doi. org/10.1002/hep.26687.

5. Dong T, Aronsohn A, Gautham Reddy K, Te HS. Rifaximin decreases the incidence and severity of acute kidney injury and hepatorenal syndrome in cirrhosis. Dig Dis Sci. 2016; 61: 3621-6. https://doi.org/10.1007/s10620-016-4313-0.

6. Arroyo V. Microalbuminuria, systemic inflammation, and multiorgan dysfunction in decompensated cirrhosis: evidence for a nonfunctional mechanism of hepatorenal syndrome. Hepatol Int. 2017; 11: 242-4. https://doi. org/10.1007/s12072-017-9784-3.

7. Nazar A, Pereira GH, Guevara M, Martin-Llahi M, Pepin MN, Marinelli M, Sola E, Baccaro ME, Terra C, Arroyo V, Gines P. Predictors of response to therapy with terlipressin and albumin in patients with cirrhosis and type 1 hepatorenal syndrome. Hepatology. 2010; 51: 219-26. https://doi.org/10.1002/hep.23283.

8. de Andrade CA, de Andrade GM, De Paula PM, De Luca LA Jr, Menani JV. Involvement of central alpha1adrenoceptors on renal responses to central moxonidine and alpha-methylnoradrenaline. Eur J Pharmacol. 2009; 607: 60-7.

9. Cai CX, Maddukuri G, Jaipaul N, Zhang Z. A treat-to-target concept to guide the medical management of hepatorenal syndrome. Dig Dis Sci. 2015; 60: 1474-81. https://doi. org/10.1007/s10620-014-3483-X.

10. Salerno F, Navickis RJ, Wilkes MM. Albumin treatment regimen for type 1 hepatorenal syndrome: a dose-response 
meta-analysis. BMC Gastroenterol. 2015; 15: 167. https:// doi.org/10.1186/s12876-015-0389-9.

11. Deshpande P, Rausa K, Turner J, Johnson M, Golestaneh L. Acute kidney injury as a causal factor in mortality associated with hepatorenal syndrome. Hepatol Int. 2011; 5: 751-8. https://doi.org/10.1007/s12072-011-9269-8.

12. Wu Z, Kong $\mathrm{X}$, Zhang $\mathrm{T}$, Ye J, Fang $\mathrm{Z}$, Yang $\mathrm{X}$. Pseudoephedrine/ephedrine shows potent anti-inflammatory activity against TNF-alpha-mediated acute liver failure induced by lipopolysaccharide/D-galactosamine. Eur J Pharmacol. 2014; 724: 112-21. https://doi.org/10.1016/j. ejphar.2013.11.032.

13. Banu S, Bhaskar B, Balasekar P. Hepatoprotective and antioxidant activity of Leucas aspera against D-galactosamine induced liver damage in rats. Pharm Biol. 2012; 50: 1592-5. https://doi.org/10.3109/13880209.2012. 685130 .

14. Hocher B, Heiden S, von Websky K, Rahnenfuhre J, Kalk P, Pfab T. Dual endothelin-converting enzyme/neutral endopeptidase blockade in rats with D-galactosamineinduced liver failure. Eur J Med Res. 2011; 16: 275-9.

15. Pushpavalli G, Kalaiarasi P, Veeramani C, Pugalendi KV. Effect of chrysin on hepatoprotective and antioxidant status in D-galactosamine-induced hepatitis in rats. Eur J Pharmacol. 2010; 631: 36-41. https://doi.org/10.1016/j. ejphar.2009.12.031.

16. Jaishree V, Badami S. Antioxidant and hepatoprotective effect of swertiamarin from Enicostemma axillare against D-galactosamine induced acute liver damage in rats. J Ethnopharmacol. 2010; 130: 103-6. https://doi. org/10.1016/j.jep.2010.04.019.

17. Pushpavalli G, Veeramani C, Pugalendi KV. Influence of chrysin on hepatic marker enzymes and lipid profile against D-galactosamine-induced hepatotoxicity rats. Food Chem Toxicol. 2010; 48: 1654-9. https://doi.org/10.1016/j. fct.2010.03.040.

18. Gueutin V, Meftah A, Desbuissons G, Debchi L, Langlois AL, Shehwaro N, Izzedine H. [Hepatorenal syndrome: focus]. [Article in French]. Nephrol Ther. 2013; 9: 471-80. https://doi.org/10.1016/j.nephro.2013.05.002.

19. Nazar A, Guevara M, Sitges M, Terra C, Sola E, Guigou C, Arroyo V, Gines P. LEFT ventricular function assessed by echocardiography in cirrhosis: relationship to systemic hemodynamics and renal dysfunction. J Hepatol. 2013; 58: 51-7. https://doi.org/10.1016/j.jhep.2012.08.027.

20. Leung N, Croatt AJ, Haggard JJ, Grande JP, Nath KA. Acute cholestatic liver disease protects against glycerol-induced acute renal failure in the rat. Kidney Int. 2001; 60: 1047-57. https://doi.org/10.1046/j.1523-1755.2001.0600031047.x.

21. Li YF, Cao XJ, Bai XY, Lin SP, Shi ST. Change of expression of renal alphal-adrenergic receptor and angiotensin II receptor subtypes with aging in rats. Aging Clin Exp Res. 2010; 22: 123-8.

22. Docherty JR. Subtypes of functional alpha1-adrenoceptor. Cell Mol Life Sci. 2010; 67: 405-17. https://doi. org/10.1007/s00018-009-0174-4.

23. Abdel Salam OM, Sleem AA, Shafee N. Hepatoprotective effects of the nitric oxide donor isosorbide-5-mononitrate alone and in combination with the natural hepatoprotectant, silymarin, on carbon tetrachloride-induced hepatic injury in rats. Inflammopharmacology. 2010; 18: 87-94. https://doi. org/10.1007/s10787-009-0027-7.

24. Yan L, Tan X, Chen W, Zhu H, Cao J, Liu H. Enhanced vasoconstriction to alpha1 adrenoceptor autoantibody in spontaneously hypertensive rats. Sci China Life Sci. 2014; 57: 681-9. https://doi.org/10.1007/s11427-014-4672-8.

25. Orallo F, Garcia-Ferreiro $\mathrm{T}$, Enguix MJ, Tristan $\mathrm{H}$, Masaguer C, Ravina E, Cadavid I, Loza MI. In vivo and in vitro pharmacological studies of a new hypotensive compound (QF0301B) in rat: comparison with prazosin, a known alpha1-adrenoceptor antagonist. Vascul Pharmacol. 2003; 40: 97-108.

26. Vivas NM, Giraldo J, Tabernero A, Vila E, Badia A. Use of the operational model of agonism and $[3 \mathrm{H}]$ prazosin binding to assess altered responsiveness of alpha1-adrenoceptors in the vas deferens of spontaneously hypertensive rat. Naunyn Schmiedebergs Arch Pharmacol. 1997; 356: 383-91. 